\section{Selecting an Antiretroviral Regimen for Human Immunodeficiency Virus Postexposure Prophylaxis in the Occupational Setting}

To the Editor-We read with great interest the recently updated US Public Health Service Guidelines for human immunodeficiency virus (HIV) postexposure prophylaxis (PEP) after occupational exposures. ${ }^{1}$ Key changes in the document, compared with previous 2005 guidelines from the Centers for Disease Control and Prevention, are the recommendations to no longer conduct exposure risk stratification and to instead uniformly use a 3-drug regimen-preferably tenofovir disoproxil fumarate (TDF), emtricitabine (FTC), and raltegravir-in exposed persons. The rationale provided for these changes was 4 -fold and included (1) the superior antiviral activity of 3 drugs versus 2 for the treatment of chronic HIV infection, (2) concerns about potential HIV drug resistance in source patients, (3) improved safety and tolerability of newer drugs, and (4) potential for improved adherence with regimens of lower toxicity. We wish to raise several issues for institutions to consider when reviewing their occupational PEP protocol in light of these guidelines.
First, while we agree that raltegravir is an excellent potential third agent for PEP whose tolerability is supported by published literature, ${ }^{2,3}$ it is noteworthy that tolerability and adherence data for this and other TDF/FTC-based regimens described in the guidelines that would justify their relative positions as preferred or alternative options are not discussed in the document. In particular, it remains unclear whether any potential adherence advantages of raltegravir related to tolerability might be offset by the disadvantage of twice daily dosing. We recently reviewed available data from our infectious diseases clinic for patients initiating TDF/FTC with lopinavir/ritonavir as either occupational or nonoccupational PEP between January 11, 2011, and May 31, 2013. Preliminary findings along with those from other published reports using a TDF/FTC backbone are summarized in Table 1 and suggest roughly comparable outcomes. ${ }^{2-4}$ Further, while we agree that raltegravir-based PEP regimens would limit concerns regarding drug interactions, our data suggest that significant polypharmacy is uncommon in most PEP users. We suggest that additional data on clinically relevant outcomessuch as regimen completion, tolerability, and PEP failuresbe systematically reported by large PEP programs wherever possible in order to inform future practice in a more evidence-based fashion. The results of ongoing clinical trials (http://www.clinicaltrials.gov) examining lopinavir/ritonavir,

TA B LE 1. Clinical Outcomes of Tenofovir Disoproxil Fumarate/Emtricitabine-Based, 3-Drug Postexposure Prophylaxis (PEP) Regimens

\begin{tabular}{|c|c|c|c|c|}
\hline & \multicolumn{2}{|c|}{ Raltegravir } & \multicolumn{2}{|c|}{ Lopinavir/ritonavir } \\
\hline & Boston $(n=100)^{2}$ & Sydney $(n=91)^{3}$ & France $(n=249)^{4}$ & Toronto $(n=124)$ \\
\hline \multicolumn{5}{|l|}{ Participant disposition } \\
\hline Completed prescribed regimen & $57(57.0)$ & $79(86.8)$ & $166(66.7)$ & $42(33.9)$ \\
\hline Switched regimen & $27(27.0)$ & & & \\
\hline Because of adverse events & NR & $1(1.1)$ & 0 & $5(4.0)$ \\
\hline Other reasons & NR & 0 & 0 & $28(22.6)$ \\
\hline Stopped because of adverse events & 0 & $1(1.1)$ & $22(8.8)$ & $1(0.8)$ \\
\hline Stopped for other reasons $s^{\mathrm{a}}$ & $1(1.0)$ & $6(6.6)$ & $34(13.7)$ & $13(10.5)$ \\
\hline Follow-up at another facility & 0 & 0 & 0 & $16(12.9)$ \\
\hline Lost to follow-up ${ }^{b}$ & $15(15.0)$ & $4(4.4)$ & $27(10.8)$ & $19(15.3)$ \\
\hline \multicolumn{5}{|l|}{ Reported side effects ${ }^{c}$} \\
\hline Nausea/vomiting & $27(27.0)$ & $22(24.2)$ & $54(21.7)$ & $66(38.6)$ \\
\hline Diarrhea & $21(21.0)$ & $23(25.3)$ & $72(28.9)$ & $69(40.4)$ \\
\hline Headache & $15(15.0)$ & $14(15.4)$ & $35(14.0)$ & $16(9.4)$ \\
\hline Fatigue/asthenia & $14(14.0)$ & $34(37.4)$ & $72(28.9)$ & $40(23.4)$ \\
\hline Muscle-related events ${ }^{\mathrm{d}}$ & NR & $8(8.8)$ & $1(0.4)$ & $7(4.1)$ \\
\hline
\end{tabular}

NOTE. Data are no. (\%). NR, not reported.

a Reasons include PEP not indicated, baseline human immunodeficiency virus seropositivity, failed eligibility.

b Includes any patient not known to have completed all 28 days of prophylaxis.

c For consistency across studies, reported as a proportion of the total number studied.

d Eight patients reporting myalgias in the Sydney study, 1 case of rhabdomyolysis in the French study, 7 patients reporting myalgias in the Toronto study. 
darunavir/ritonavir, raltegravir, and maraviroc in various randomized comparisons will allow for more evidence-based selection of PEP regimens in the future.

Second, epidemiologic evidence of increasing transmitted resistance is cited in the guidelines as a rationale for uniformly recommending the use of 3 drugs. ${ }^{5}$ Given this concern, however, a relevant disadvantage of raltegravir is its modest genetic barrier to resistance. In the SWITCHMRK trials, adults with well-controlled HIV infection on a lopinavir/ritonavirbased regimen maintained virologic suppression of fewer than 50 copies $/ \mathrm{mL}$ at 24 weeks less often when switched to raltegravir compared with those who continued their original regimen (difference, $-6.2 \% ;-11.2 \%$ to $-1.3 \%$ ). According to the study authors, this finding was likely driven by decreased efficacy in the setting of underlying resistance to nucleoside reverse transcriptase inhibitors (NRTIs). ${ }^{6}$ Extrapolation to the PEP setting suggests that caution is warranted with this regimen if a source patient is known or suspected to harbor NRTI-resistant virus.

Third, we note that the recommendation to remove the risk stratification step offers the programmatic advantage of simplifying clinical decision making regarding how many drugs to prescribe and may thus be particularly advantageous for healthcare providers who encounter this scenario uncommonly. As such, it may be particularly applicable to cases of nonoccupational PEP (eg, for consensual sexual exposures), which are more often assessed in busy emergency departments staffed by large numbers of rotating personnel and for which studies have previously documented inconsistent practices. ${ }^{78}$ However, this advantage may be less relevant to occupational PEP, which is often handled in institutional corporate health clinics by limited numbers of expert occupational healthcare providers. To the extent that risk assessment must already be performed to determine whether PEP is indicated, the incremental benefit of eliminating further risk stratification is debatable.

Finally, a major consideration not discussed in the guidelines is medication cost. In our jurisdiction, the incremental cost of adding a 28-day course of twice-daily raltegravir to a 2-drug regimen of TDF/FTC is $\$ 756$ Canadian dollars per case. In a recent review of all 161 reported occupational blood/body fluid exposures seen at our institutional corporate health department between July 23, 2012, and August 21, 2013, PEP was administered to 41 staff, of whom 29 received TDF/FTC; adding raltegravir would have cost an additional $\$ 21,924$ over this 13 -month period. This added cost may be justifiable if it averted even a single case of HIV transmission, but numerical estimates on the preventive efficacy of modern $P E P$ regimens is and, for ethical reasons, will remain lacking. The relative rarity of PEP failure both anecdotally and in the published literature suggests that 2-drug regimens are likely to be adequate in many cases. PEP programs should be encouraged to publish their experience to better inform the evidence base for this important preventive health intervention.

In summary, while the recommendations in the revised occupational PEP guidelines may offer some advantages over current practice, more data are needed to help institutions justify the choices of preferred and alternative regimens in an evidence-based fashion, and issues such as drug resistance and cost must be given careful consideration.

\section{ACKNOWLEDGMENTS}

Financial support. This work was funded by the Ontario Ministry of Health and Long-Term Care Academic Funding Plan Innovation Fund.

Potential conflicts of interest. D.H.S.T. reports that he has received research grants from Gilead Sciences Canada and Viiv Healthcare; has received speaking honoraria from Abbott, Bristol-Myers Squibb, Gilead, Janssen, Merck, and Viiv; and is supported by a Randomized Controlled Trials Mentoring Award from the Canadian Institutes of Health Research. All other authors declare no conflicts of interest relevant to this article. All authors submitted the ICMJE Form for Disclosure of Potential Conflicts of Interest, and the conflicts that the editors consider relevant to this article are disclosed here.

Darrell H. S. Tan, MD, PhD; ${ }^{1,2}$ Blessing Goddey-Erikefe, BSc; ${ }^{1}$ Deborah Yoong, PharmD; ${ }^{1}$ Mark Naccarato, BScPharm; ${ }^{1}$ Susan McIntyre, RN, COHN(C), CRSP; ${ }^{3}$ Roohi Qureshi, $\mathrm{MD}_{;}^{3}$ Kevin Gough, $\mathrm{MD}^{1,2}$

Affiliations: 1. Division of Infectious Diseases, St. Michael's Hospital, Toronto, Ontario, Canada; 2. Division of Infectious Diseases, University of Toronto, Toronto, Ontario, Canada; 3. Corporate Health and Safety, St. Michael's Hospital, Toronto, Ontario, Canada.

Address correspondence to Darrell H. S. Tan, MD, FRCPC, 30 Bond Street, 4CC, Room 4-179, Toronto, Ontario M5B 2W9, Canada (darrell.tan@ gmail.com).

Infect Control Hosp Epidemiol 2014;35(3):326-328

(C) 2014 by The Society for Healthcare Epidemiology of America. All rights reserved. 0899-823X/2014/3503-0022\$15.00. DOI: 10.1086/675297

\section{REFERENCES}

1. Kuhar DT, Henderson DK, Struble KA, et al. Updated US Public Health Service guidelines for the management of occupational exposures to human immunodeficiency virus and recommendations for postexposure prophylaxis. Infect Control Hosp Epidemiol 2013;34:875-892.

2. Mayer KH, Mimiaga MJ, Gelman M, Grasso C. Raltegravir, tenofovir DF, and emtricitabine for postexposure prophylaxis to prevent the sexual transmission of HIV: safety, tolerability, and adherence. J Acquir Immune Defic Syndr 2012;59:354-359.

3. McAllister J, Read P, McNulty A, Tong W, Ingersoll A, Carr A. Raltegravir-emtricitabine-tenofovir as HIV nonoccupational post-exposure prophylaxis in men who have sex with men: safety, tolerability and adherence. HIV Med 2014;15:13-22.

4. Tosini W, Muller P, Prazuck T, et al. Tolerability of HIV postexposure prophylaxis with tenofovir/emtricitabine and lopinavir/ ritonavir tablet formulation. AIDS 2010;24:2375-2380.

5. Wheeler WH, Ziebell RA, Zabina $\mathrm{H}$, et al. Prevalence of trans- 
mitted drug resistance associated mutations and HIV-1 subtypes in new HIV-1 diagnoses, U.S.-2006. AIDS 2010;24:1203-1212.

6. Eron JJ, Young B, Cooper DA, et al. Switch to a raltegravir-based regimen versus continuation of a lopinavir-ritonavir-based regimen in stable HIV-infected patients with suppressed viraemia (SWITCHMRK 1 and 2): two multicentre, double-blind, randomised controlled trials. Lancet 2010;375:396-407.

7. Barnes S, Loutfy M, Prasad R, Tan DHS. Institutional policies and physician prescribing practices of non-occupational postexposure prophylaxis (nPEP) in Ontario. In: 21st Canadian Conference on HIV/AIDS Research; 2012; Montreal, Canada. Abstract P158.

8. Kunches LM, Meehan TM, Boutwell RC, McGuire JF. Survey of nonoccupational HIV postexposure prophylaxis in hospital emergency departments. I Acquir Immune Defic Syndr 2001;26:263265.

\section{Reply to Tan et al}

To the Editor-In the letter by Tan et al, ${ }^{1}$ in response to the updated US Public Health Service (PHS) guidelines, ${ }^{2}$ several issues are raised for consideration by institutions when developing their protocols for occupational exposures to human immunodeficiency virus (HIV). We emphasize that the US PHS guidelines are not intended to be used as a strict protocol; they are open to interpretation and modification, based on local circumstances. The PHS working group and expert consultant panel used available scientific evidence and expert opinion as the basis for developing the updated guidelines. However, evidence of superior efficacy of a single PEP regimen among the preferred and alternatives ${ }^{2}$ does not exist and is unlikely to be developed. Demonstrating differential efficacy among PEP regimens that likely possess a similar ability to prevent infection is limited by both the low HIV transmission rate associated with occupational exposures as well as the ethical considerations associated with conducting a randomized controlled trial in that setting. Thus, most of the opinion expressed in the guideline was based on relevant but indirect evidence. The expert panel believed that the regimen adherence advantages of a raltegravir (RAL)-based regimen offered a slight benefit over similar regimens containing protease inhibitors. An optimal single PEP regimen for occupational exposures has not been demonstrated and, given the constraints noted above, likely never will be.

We agree that evidence of PEP tolerability and adherence are among the factors that should inform PEP regimen choices. Tan et al $^{1}$ describe clinical outcome data ${ }^{1,3-5}$ among RAL- and lopinivir/ritonavir-based PEP regimen recipients who primarily experienced nonoccupational exposures. Though the authors suggest roughly comparable outcomes, we interpret these data differently. The $15 \%$ higher average regimen completion rate among RAL-based PEP regimen recipients seems to indicate a slight advantage of RAL-based
PEP. We nonetheless recommend caution when extrapolating from data describing primarily nonoccupational PEP recipients to the occupational setting. Historically, healthcare personnel taking occupational PEP have reported much higher rates of regimen intolerance than persons taking these agents for either nonoccupational exposures or as treatment for infection; ${ }^{6}$ thus, one might expect different PEP completion rates between nonoccupational and occupational exposure populations.

Tan et $\mathrm{al}^{1}$ question the benefit of the minimal drug interactions afforded by RAL-based PEP regimens and indicate that significant polypharmacy is uncommon among their PEP recipients. Minimizing the risk for drug interactions can increase medication adherence and acceptance. Taking even a single medication (either prescription or over the counter) while receiving PEP can place a PEP recipient at risk for significant drug interactions. Because RAL can be administered with proton pump inhibitors, $\mathrm{H} 2$ blockers, antidepressants, and oral contraceptives, all of which are commonly used by relatively healthy personnel, we believe that RALbased regimens might have a relative advantage. PEP regimen adherence rarely exceeds $85 \%$ in most published studies, suggesting that adherence remains a significant issue. Thus, addressing factors that can improve adherence is likely to increase effectiveness.

The commentary authors suggest caution with the use of the tenofovir, emtricitabine, and RAL regimen as PEP for exposures to source patients known or suspected to harbor viruses resistant to nucleotide reverse-transcriptase inhibitors. We agree-and the guidelines indicate-that special considerations should be given to circumstances in which exposure to resistant virus is likely. Expert consultation is recommended for exposures to known or suspected drug-resistant HIV to ensure that drugs to which the source virus is unlikely to be resistant are prescribed as PEP. ${ }^{2}$ The relevance of RAL's modest genetic barrier to resistance in the treatment of HIV infection may not be directly applicable to the success of PEP. PEP efficacy data remain too limited to indicate whether or how genetic barriers to resistance influence HIV PEP outcomes.

Tan et $\mathrm{al}^{1}$ question whether simplification of clinical decision making by eliminating exposure risk stratification may be less relevant to occupational PEP and suggest that occupational exposures are often managed in institutional corporate health clinics by expert occupational health providers. Occupational health clinics may provide management for exposures that occur in outpatient and inpatient settings when exposures occur during the daytime hours during which occupational health clinics are typically open. However, such occupational health clinics are unlikely to be available for individuals sustaining exposures outside these normal clinic hours. For facilities that provide 24 -hour patient care-such as acute care hospitals, long-term acute care hospitals, skilled nursing facilities, and emergency treatment centers-occupational exposures to bloodborne pathogens occur at all 